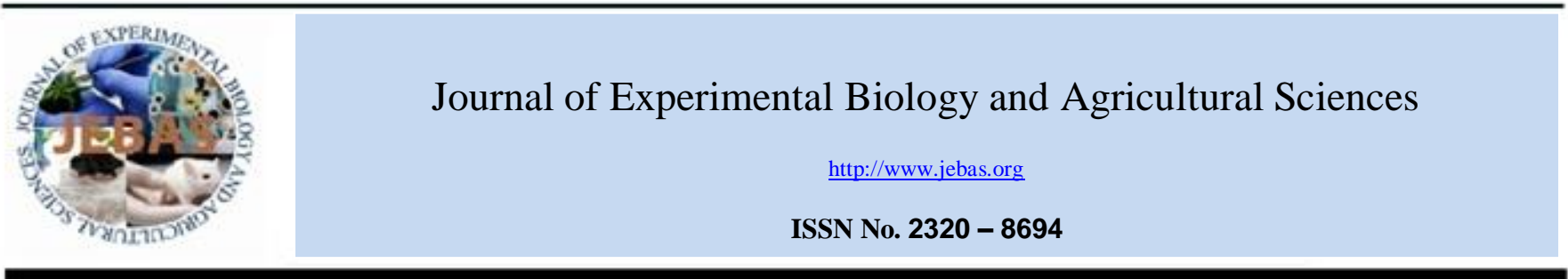

\title{
ISOLATION OF HETEROTROPHIC MICROALGAE FROM HOT SPRINGS FOR LIPID PRODUCTION
}

\section{Basil D’Mello $^{1,2 *}$, Maya Chemburkar ${ }^{1}$}

\author{
${ }^{1}$ Department of Botany, Bhavan's College, Andheri(W), Mumbai-400058 \\ ${ }^{2}$ Department of Biotechnology, VIVA College, Virar (W), Maharashtra 401303 \\ Received - November 04, 2018; Revision - December 05, 2018; Accepted - December 10, 2018 \\ Available Online - December 15, 2018
}

DOI: http://dx.doi.org/10.18006/2018.6(6).1010.1014

KEYWORDS
Auxenochlorella pyrenoidosa
Heterotrophic
Lipid
Biodiesel
Hot springs.

\begin{abstract}
Biodiesel is an alternative to diesel fuel, which is produced from bio oils via transesterification. It is nontoxic, biodegradable and has the potential to replace the conventional diesel fuel. Microalgae can produce fatty acids called unsaturated lipids, which can be extracted and processed to form biofuel. The present study was aimed to isolate of heterotrophic microalgae from hot springs of Palghar, Maharashtra for lipid production. Auxenochlorella pyrenoidosa was isolated and tested for its lipid production ability. Results of study revealed that $A$. pyrenoidosa has the ability to produce about $11.56 \%$ lipid of its dry weight. Fatty acid profile of the extracted lipid showed the presence of C14:0, C16:0, C18:0, C16:1, C18:1, C18:2 and C18:3 fatty acids which make it a good biodiesel feedstock.
\end{abstract}

* Corresponding author

E-mail: dmello.basil@gmail.com (Mr. Basil D’Mello)

Peer review under responsibility of Journal of Experimental Biology and Agricultural Sciences.

Production and Hosting by Horizon Publisher India [HPI] (http://www.horizonpublisherindia.in/).

All rights reserved.
All the article published by Journal of Experimental Biology and Agricultural Sciences is licensed under a Creative Commons Attribution-NonCommercial 4.0 International License Based on a work at www.jebas.org.

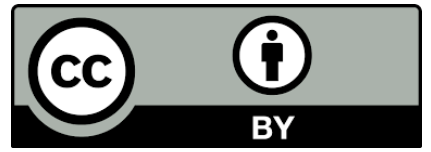




\section{Introduction}

Biodiesel is produced by transesterification of oils and has the capability of being an alternative for the conventional diesel fuel. Also it is nontoxic and biodegradable. The use of biodiesel will ultimately lead to a reduction of harmful emissions of carbon monoxide, hydrocarbons and particulate matter and to the elimination of SOx emissions, which can also help in reducing the greenhouse effects and global warming (Khan et al., 2009; Singh \& Singh, 2010; Mahmudul et al., 2017).

Microalgae are small sized organisms which can grow in fresh water and marine ecosystems, have been gaining a lot of interest as a biodiesel feedstock. Microalgae manufacture and store lipids within their cells, especially under stress environment. Under stress conditions like nitrogen starvation, microalgal growth is inhibited and the cells store lipids as a survival strategy. These storage lipids can be converted to biodiesel (Liu et al., 2017; Tan et al., 2017). Algae have capability to produce at least 15 times more oil per acre than alternatives such as rapeseed, palms, soybeans, or jatropha (Parmar et al.,2011; Chen et al., 2018). Moreover, algae-growing facilities can be built on land unsuitable for conventional agriculture. They can grow 20 to 30 times faster than food crops. The hard part about algae production is growing the algae in a controlled way and harvesting it efficiently (Voloshin et al., 2016).

Local microalgae species can be screened for lipid production as it can be expected that they are better adapted for the resident geographical, climatic and ecological conditions over laboratory strains (Hu et al., 2008; Mata et al., 2010). Most organisms have evolved under relatively typical climates and are not normally able to survive in extreme environment such as temperature, $\mathrm{pH}$ etc. However, there are areas on earth where environmental conditions are beyond the normal limits for growth and can thus be considered as extreme. A wide range of microalgal species worldwide are found in such extreme environments (Varshney et al., 2015). In the present study, green microalgae of Chlorella spps was isolated from a hot spring and tested for its lipid productivity and its potential use of the fatty acids in production of Biodiesel.

\section{Materials and Methods}

\subsection{Isolation of Heterotrophic Microalgae}

Water samples were collected from Sativali hot springs located in Palghar District, Maharashtra. The water samples were plated on BG 11 agar plates (Himedia). Green colonies were further purified on BG 11 agar plates to obtain pure culture. The cultured microalgae were assessed microscopically for contamination. The microalgal isolate was also inoculated on BG11 agar supplemented with $0.1 \%$ glucose and incubated in dark to check for heterotrophic growth.

\subsection{Molecular Identification of Microalgae:}

The molecular identification of the isolated microalgae was carried out by Xcelris Labs Ltd, Ahmedabad, India. The DNA was isolated from the culture using Xcelgen gDNA kit. Isolated DNA was amplified with 18S rRNA Specific Primer (1F and 4R) using Veriti ${ }^{\circledR} 96$ well Thermal Cycler (Model No. 9902). A single discrete PCR amplicon band of 900 bp was observed. The PCR amplicon was enzymatically purified and further subjected to Sanger sequencing. Bi-directional DNA sequencing reaction of PCR amplicon was carried out with $1 \mathrm{~F}$ (CTGGTGCCAGCAGCCGCGGYAA) and 4R(CKRAGGGCATYACWGACCTGTTAT) primers using BDT v3.1 Cycle sequencing kit on ABI 3730xl Genetic Analyzer.The $18 \mathrm{~S}$ gene in SSU region sequence was used to carry out BLAST alignment search tool of NCBI genbank database.

\subsection{Effect of Autotrophic and Heterotrophic mode of nutrition on Biomass and lipid production}

The microalgal isolate was inoculated in $100 \mathrm{ml}$ of BG 11 and cultured for 14 days. The microalgae cells were separated by centrifugation and then were inoculated in separate flasks containing $50 \mathrm{ml}$ of BG 11 broth with and without Sodium Nitrate (Nitrogen source). The Culture was grown at $25^{\circ} \mathrm{C}$, with approximately 8000 lux white light illumination and 16 :8 hours light: dark photoperiod on an orbital shaker at $100 \mathrm{rpm}$ for 7 days

\subsection{Extraction of Lipid and Fatty Acid Methyl Ester (FAME) analysis}

A $20 \mathrm{ml}$ sample of the microalgal broth is centrifuged. The biomass pellet is recovered and extracted with chloroform/methanol/water system (1:1:0.9) as described by Işik et al. (1999). The mass of the lipids extracted is measured gravimetrically by evaporating the solvent. The extracted lipid was subjected to transesterification by Metcalfe et al. (1966) in which the sample containing lipid fraction was placed in a test tube where a mixture $3 \mathrm{~mL}$ of $\mathrm{BF}_{3} / \mathrm{MeOH}$ was added. The mixture was heated in a water bath at $70^{\circ} \mathrm{C}$ for $20 \mathrm{~min}$. For recovery of the fatty acid methyl esters the derivatized mixture was washed into a separatoryfunnel with $15 \mathrm{~mL}$ of hexane and 20 $\mathrm{mL}$ of distilled water.

The organic and aqueous phases were then separated. The organic phase containing the fatty esters was dried, and the solvent was evaporated at $50^{\circ} \mathrm{C}$ by GC/MS analyses using an Agilent $7890 \mathrm{~A}$ gas-chromatograph equipped with a 8975C MS detector and fitted with an HP-5 MS fused silica column (length $30 \mathrm{~m}$; i.d. 0.25 $\mathrm{mm}$; film thickness $0.25 \mathrm{~mm}$ ). 


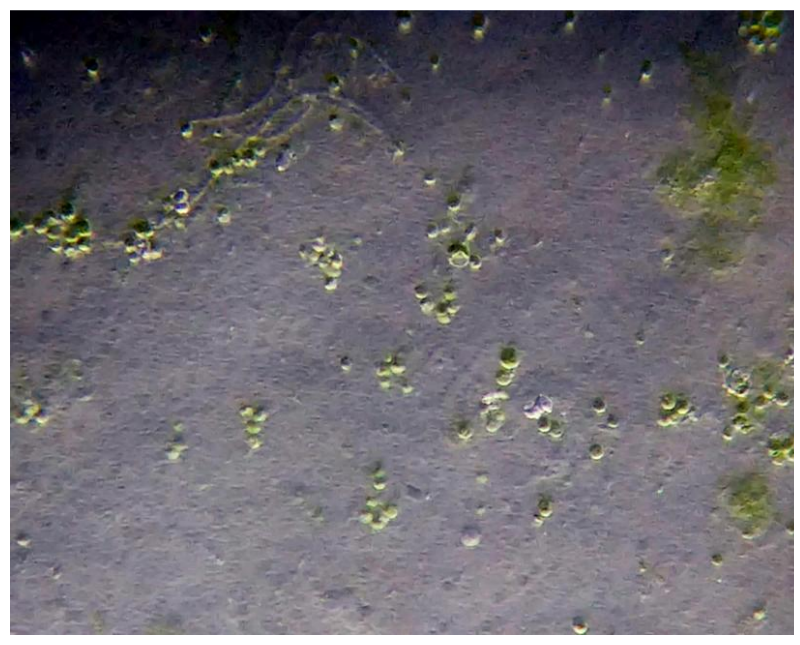

Figure 1 Auxenochlorella pyrenoidosa (400X)

3 Results

\subsection{Isolation of Microalgae}

After inoculation of the water sample, considerable growth was observed on the plates after 7 days. Each Green Colony was subcultured on sterile BG11 medium. Serial subculturing of the microalgae was done to obtain axenic cultures. On Microscopic examination, the microalgae cells were observed to be spherical in shape and green in colour. No flagella were observed. The cells were present singly and also in clumps. The isolated microalga wasable to grow on BG11 medium supplemented with $0.1 \%$ glucose which indicated that it was capable of growing heterotrophically. Purity of the culture was verified microscopically and no contaminating microalgae species were found.

\subsection{Molecular Identification of Microalgae}

The DNA was isolated from the culture and was amplified with 18S rRNA Specific Primer (1F and 4R). The PCR amplicon was enzymatically purified and further subjected to Sanger Sequencing. After sequencing the sequence was found to be matching to A. pyrenoidosa.

\subsection{Biomass and Lipid production}

The microalgal isolates were subjected to nitrogen deprivation for lipid accumulation. There was a drastic difference in lipid production when the nitrogen concentration was limited under autotrophic mode. In heterotrophic mode, the lipid content increased but not to a great extent. During nitrogen limitation or starvation the metabolic flux is shifted from protein synthesis to lipid production and hence the lipid concentration increases.

\subsection{FAME analysis of extracted Lipids}

The FAME analysis of the extracted lipids after transesterification showed the presence of C14:0, C16:0, C18:0, C16:1, C18:1, C18:2 and C18:3. Differences were observed in the fatty acid profiles of the microalgae when cultured under autotrophic mode and heterotrophic mode.

\section{Discussions and Conclusions}

The availability of organic carbon and nitrogen deprivation are crucial for efficient lipid productivity in microalgae (Liu et al. 2013; Babuskin et al. 2014). It has been reported that for $C$. Pyrenoidosa maximum biomass production was achieved when glucose was supplied in the medium as a carbon source (Ratnapuram et al., 2018). In the present study, the presence or absence of nitrogen and organic carbon in the culture medium concurrently influenced lipid accumulation inthe isolated

TTTCTTATCCATACCGTAATTTAAAAGTTTGGTTGCAGTTAAAAGCTCGTAGTTGGATTTCGGAAGGTCCTTAGCAGTCC GCCCCTTCGGGGAGCGGGTTGCTGGCCTCCTATGTTCCTAACGGTCCTCATCCGCGAGGGTGGGGAATCAACCGCTAGGA TCGTTTACTTTGAGGAAATTAGAGTGTTCAAAGCAGGCGTAACTCGCCTCCGAATACGTTAGCATGGGATAATGGAATAC GACTTCGGTCTTGTTTCGTTGGTTTCGCTTGGCTGAAGTAATGATTGATAGGGACAGTTGGGGGCATTAGTATTTAGTTG TCAGAGGTGAAATTCTAGGATTTACTAAAGACTGACCAATGCGAAAGCATTTGCCAAGGATGTTTTCATTAATCAAGAAC GAAAGTAGGGGGATCGAAGACGATCAGATACCGTCGTAGTCTCTACCGTAAACTATGCCGACCAGGGATCCGGAGAGTTT GCATGGATGACTCCCCGGGCACCTTGTGAGAAATCATAAGTGTTTGGGTTTTGGGGGGAGTATGGTCGCAAGGCTGAAAC TTAAAGGAATTGACGGAAGGGCACCACCAGGAGTGGAACCTGCGGCTTAATTTGACTCAACACGGGGAAACTTACGAGGT CAGGACACTGTGAGGATTGACAGATTGAAAGCTCTTTCTTGATTCAGTGGGTGGTGGTGCATGGCCGTTCTTAGTTGGTG GAGTGATTTGTCTGGTTAATTCCGTTAACGAACGAGACCTTAACCTGCTAAATAGTCACGCGAACCGGTCCGCAGGGCTC AAAGTTCGCGGCTCGACTTCTTAGAGGGACTATTCGCACCCGCCAGCGATGGAAGTTTGAGGCAATAACAGGTCAGTAGT GTGCCCTAAGAAT

Figure 218S rRNA sequence of Auxenochlorella pyrenoidosa

Journal of Experimental Biology and Agricultural Sciences http://www.jebas.org 
Table 1 Lipid production in A. pyrenoidosa under autotrophic and heterotrophic modes of nutrition

\begin{tabular}{|c|c|c|c|c|c|}
\hline & Name & Mode & Condition & Dry cell weight $(\mathrm{mg} / \mathrm{L})^{\mathrm{a}}$ & $\%$ of lipid extracted \\
\hline \multirow{4}{*}{ A. } & \multirow{4}{*}{ pyrenoidosa } & \multirow{2}{*}{ Autotrophic } & With Nitrogen source & $1196 \pm 20$ & 4.6 \\
\hline & & & Without Nitrogen source & $988 \pm 26$ & 9.3 \\
\hline & & \multirow{2}{*}{ Heterotrophic } & With Nitrogen source & $1068 \pm 16$ & 10.53 \\
\hline & & & Without Nitrogen source & $864 \pm 13$ & 11.56 \\
\hline
\end{tabular}

${ }^{\mathrm{a}}$ Mean $\pm \operatorname{SD}(\mathrm{n}=3)$

Table 2 Fatty acid profile of A. pyrenoidosa under autotrophic and heterotrophic modes of nutrition

\begin{tabular}{|c|c|c|}
\hline \multirow{2}{*}{ Fatty Acids } & Under Autotrophic Mode & Pyrenoidosa \\
\hline Saturated Fatty Acids & $5.07 \%$ & $13.39 \%$ \\
\hline C14:0 & $0.62 \%$ & $2.32 \%$ \\
\hline C16:0 & $3.6 \%$ & $10.78 \%$ \\
\hline C18:0 & $0.85 \%$ & $0.29 \%$ \\
\hline Monosaturated Fatty Acids & $1.1 \%$ & $8.33 \%$ \\
\hline C16:1 & $0.41 \%$ & $5.88 \%$ \\
\hline C18:1 & $0.77 \%$ & $2.45 \%$ \\
\hline Polyunsaturated Fatty acids & $10.67 \%$ & $25.88 \%$ \\
\hline C18:2 & $1.88 \%$ & $4.13 \%$ \\
\hline C18:3 & $8.79 \%$ & $21.75 \%$ \\
\hline
\end{tabular}

microalgae. Under unfavourable conditions especially nitrogen deficiency; microalgae accumulate more lipid as energy storage (Pal et al. 2011; Huang et al., 2013). However, the remarkable increase of algal lipid content in this study was found in the treatment without nitrogen addition. For commercial lipid production, algae need to have high biomass productivity and high lipid content (Lv et al., 2010). In previous studies, it was found that supplementation of organic carbon in the culture medium enhanced algal biomass production but the lipid content in algae was not affected (Heredia-Arroyo et al. 2011; Cheirsilp \& Torpee 2012; Babuskin et al. 2014). Similar results were observed in the current study as well.

The present study shows that supplementation of organic carbon in the medium affects the saturated fatty acid and monounsaturated fatty acid content in the microalgae but the polyunsaturated fatty acid content is affected by presence of both organic carbon and nitrogen in the medium. The effect of nitrogen deprivation/starvation on the growth of microalgae was studied under autotrophic and heterotrophic conditions. Growth was enhanced in heterotrophy compared with that in autotrophy, but the growth advantage disappeared under nitrogen starvation. This study shows that the algae grow faster under heterotrophic conditions than autotrophic conditions, while the requirements for $\mathrm{N}$ are similar between the two modes of nutrition.

The fatty acid profile of A. Pyrenoidosa contained major fatty acids that traditional biodiesel feedstock possesses with the high content of $\mathrm{C} 16: 1$, which is considered to be the best fatty acid to improve biodiesel's characteristics after C18:1. A. Pyrenoidosa was very productive under both autotrophic and heterotrophic mode of nutrition and was found to be the best candidate for the production of biodiesel. Further detail studies are required to determine if the isolated strains can be optimised in terms of media composition and culture conditions for better productivity.

\section{Conflict of Interest}

Authors would hereby like to declare that there is no conflict of interests that could possibly arise.

\section{References}

Babuskin S, Radhakrishnan K, Babu PAS, Sivarajan M, Sukumar M (2014) Effect of photoperiod, light intensity and carbon sources on biomass and lipid productivities of Isochrys isgalbana. Biotechnology Letters 36: 1653-1660. 
Cheirsilp B, Torpee S (2012) Enhanced growth and lipid production of microalgae under mixotrophic culture condition: effect of light intensity, glucose concentration and fed-batch cultivation. Bioresource Technology110:510-516.

Chen J, Li J, Dong W, Zhang X, Tyagi RD, Drogui P, Surampalli RY (2018) The potential of microalgae in biodiesel production. Renewable and Sustainable Energy Reviews 90:336-346.

Heredia-Arroyo T, Wei W, Ruan R, Hu B (2011) Mixotrophic cultivation of Chlorella vulgaris and its potential application for the oil accumulation from non-sugar materials. Biomass and Bioenergy 35: 2245-2253.

Hu Q, Sommerfeld M, Jarvis E, Ghiradi M, Posewitz M, Seibert M, Darzins A (2008) Microalgal triacylglycerols as feed stocks for biofuel production: perspectives and advances. The Plant Journal 54: 621-639.

Huang X, Huang Z, Wen W, Yan J (2013) Effects of nitrogen supplementation of the culture medium on the growth, total lipid content and fatty acid profiles of three microalgae (Tetraselmis sub cordiformis, Nannochlorops isoculata and Pavlova viridis). Journal of Applied Phycology 25: 129-137.

Isik O, Sarihan E, Kusvuran E, Gul O, Erbatur O (1999) Comparison of the fatty acid composition of the freshwater fish larvae Tilapia zillii, the rotifer Brachionuscalyciflorus, and the microalgae Scenedesmus abundans, Monoraphidium minitum andChlorella vulgaris in the algaerotifer- fish larvae food chains. Aquaculture174: 299-311.

Khan SA, Hussain MZ, Prasad S, Banerjee UC (2009) Prospects of biodiesel production from microalgae in India. Renewable and Sustainable Energy Reviews 13: 2361-2372.

Liu J, Sommerfeld M, Hu Q (2013). Screening and characterization of Isochrysis strains and optimization of culture conditions for docosahexaenoic acid production. Applied Microbiology and Biotechnology 97: 4785-4798.

Liu J, Song Y, Qiu W (2017). Oleaginous microalgae Nannochloropsis as a new model for biofuel production: review \& analysis. Renewable and Sustainable Energy Reviews 72: 154-162.

Lv JM, Cheng LH, Xu XH, Zhang L, Chen HL (2010) Enhanced lipid production of Chlorella vulgaris by adjustment of cultivation conditions. Bioresource Technology 101: 6797-6804.

Mahmudul HM, Hagos FY, Mamat R, Adam AA, Ishak WFW, Alenezi R (2017) Production, characterization and performance of biodiesel as an alternative fuel in diesel engines-A review. Renewable and Sustainable Energy Reviews 72: 497-509.

Mata TM, Martins AA, Caetano NS (2010) Microalgae for biodiesel production and other applications: a review. Renewable and Sustainable Energy Reviews 14: 217-232.

Metcalfe LD, Schmitz AA, Pelka JR (1966) Rapid preparation of fatty acid esters from lipids for gas chromatographic analysis. Analytical Chemistry 38: 514-515.

Pal D, Khozin-Goldberg I, Cohen Z, Boussiba S (2011) The effect of light, salinity, and nitrogen availability on lipid production by Nannochloropsis sp. Applied microbiology and Biotechnology 90: 1429-1441.

Parmar A, Singh NK, Pandey A, Gnansounou E, Madamwar D (2011) Cyanobacteria and microalgae: a positive prospect for biofuels. Bioresource Technology 102: 10163-10172.

Ratnapuram HP, Vutukuru SS, Yadavalli R (2018) Mixotrophic transition induced lipid productivity in Chlorella pyrenoidosa under stress conditions for biodiesel production. Heliyon 4: e00496.

Singh SP, Singh D (2010) Biodiesel production through the use of different sources and characterization of oils and their esters as the substitute of diesel: a review. Renewable and Sustainable Energy Reviews 14: 200-216.

Tan X, Lam MK, Uemura Y, Lim JW, Wong CY, Lee KT (2017) Cultivation of microalgae for biodiesel production: a review on upstream and downstream processing. Chinese Journal of Chemical Engineering 26: 17-30.

Varshney P, Mikulic P, Vonshak A, Beardall J, Wangikar PP (2015) Extremophilic micro-algae and their potential contribution in biotechnology. Bioresource Technology 184: 363-372.

Voloshin RA, Rodionova MV, Zharmukhamedov SK, Veziroglu TN, Allakhverdiev SI (2016) Biofuel production from plant and algal biomass. International Journal of Hydrogen Energy 41: 17257-17273. 